\title{
Immunohistochemical Expression of CDC7 in Dentigerous Cyst, Odontogenic Keratocyst and Radicular Cyst
}

\author{
Zohreh Jaafari-Ashkavandi ${ }^{1}$, Ahmad Alipour Tuyeh², Sepideh Assar ${ }^{3, *}$
}

\begin{abstract}
$\mathrm{CDC7}$ is a serine/threonine kinase which has an essential role in initiation of DNA proliferation and S phase. It increases the invasion and proliferation in many pathologic lesions. This study aimed to evaluate the expression of CDC7 in the most common odontogenic cysts. We evaluated 17 dentigerous cysts, 18 odontogenic keratocysts (OKC) and 13 radicular cysts immunohistochemically. The mean expression of CDC7 was analyzed using ANOVA and Post-HOC methods. All specimens revealed CDC7 expression. Higher expression of CDC7 in OKC and radicular cyst was shown in comparison to dentigerous cyst $(P<0.001)$, while radicular cyst and OKC groups showed no difference in $C D C 7$ expression $(P=0.738)$. The high expression of $C D C 7$ in OKC suggests that this protein could be related to the higher proliferation rate and invasiveness of OKC. On the other hand, the higher $C D C 7$ expression in radicular cyst may simply be related to inflammation as this cyst is neither aggressive nor invasive.
\end{abstract}

\section{KEYWORDS}

CDC7; dentigerous cyst; odontogenic keratocyst; radicular cyst; immunohistochemistry

AUTHOR AFFILIATIONS

${ }^{1}$ Department of Oral \& Maxillofacial Pathology, Shiraz Dental School, Shiraz University of Medical Sciences, Shiraz, Iran

${ }^{2}$ Shiraz Dental School, Shiraz University of Medical Sciences, Shiraz, Iran

${ }^{3}$ Department of Oral \& Maxillofacial Pathology, Hormozgan Dental School, Hormozgan University of Medical Sciences, Hormozgan, Bandar-Abbas, Iran

* Corresponding author: Hormozgan University of Medical Sciences, Hormozgan, Bandar-Abbas, Iran; e-mail: se.assar@gmail.com

Received: 23 October 2017

Accepted: 23 March 2018

Published online: 2 July 2018

Acta Medica (Hradec Králové) 2018; 61(1): 17-21

https://doi.org/10.14712/18059694.2018.18

(c) 2018 The Authors. This is an open-access article distributed under the terms of the Creative Commons Attribution License (http://creativecommons.org/licenses/by/4.0), which permits unrestricted use, distribution, and reproduction in any medium, provided the original author and source are credited. 


\section{INTRODUCTION}

Odontogenic cysts are developmental lesions that form because of abnormalities that can occur during tooth development. These lesions are commonly found in routine radiographic examination (1). Dentigerous cyst (DC) is the most common developmental odontogenic cyst that involves about $24 \%$ of the epithelium lined cysts of the jaws $(2,3)$. This slow-growing cyst involves the crown of anunerupted tooth due to changes in the reduced enamel epithelium after completion of amelogenesis that results in aggregation of liquid between the reduced enamel epithelium and tooth crown (2-5). Odontogenic keratocyst (OKC) is one of the common cysts that accounts for about $11 \%$ of all developmental odontogenic cysts (6) originating from the remnants of the dental lamina. The OKC has a high growth rate and tendency for invasion and recurrence (7). Because of this aggressive behavior, from 2005 to 2017, the OKC was named keratocystic odontogenic tumor by the World Health Organization (WHO) $(8,9)$. In 2017 , the WHO reverted it to the odontogenic keratocyst as a cystic lesion; hence, there is a debate around the putative neoplastic nature of this lesion (9).

Radicular cyst (RC) is the most common cyst of the oral cavity which originates from the epithelium existing in the periodontal space or forming as a result of pulp necrosis (10-13). This inflammatory cyst usually has a low growth rate and is asymptomatic unless there is acute inflammation in the area (12).

In many instances, the differentiation between different cysts is difficult; thus, finding a diagnostic aid seems necessary. Todays, immunohistochemistry (IHC) using appropriate diagnostic or prognostic markers can help the pathologists.

Cell division cycle 7 (CDC7) protein is a serine/threonine kinase which plays a key role in the initiation of DNA replication and regulation of G1/S phase transition (check point of the cell cycle) (14). Different mechanisms of modulation of kinase activity in response to DNA replication stress have been reported. In one hypothesis, CDC7 plays an active role in cell cycle stages, but in another one it is an inactive final target in the cell cycle check-point $(15,16)$. It seems that overexpression of CDC7 by disabling p53 is related to several cancers and cancer cell lines (15).

Hess and colleagues found that CDC7 expresses in many normal tissues such as placenta, fetal tissues, brain, lung and immune system tissues, but it expresses further in many tumors such as colorectal carcinoma, different types of leukemia, lung carcinoma melanoma and all of their modified cell lines. They concluded that expression of CDC7 may be related to increasing proliferation of some tumors and neoplastic changes in other tumors (17). Bonte et al. studied the presence of $C D C 7$ in certain tumors and normal cells and found that CDC7 protein expression in normal tissues derived from the breast, lung and colon was very low or undetectable, but this protein was overexpressed in about $50 \%$ of 62 human tumor cells such as different types of leukemia. Overexpression of CDC7 was shown in primary tumors of the breast, colon and lung, but it was not detected in normal tissues of those areas. They also showed the association between decreasing the p53 and overexpression of CDC7 and DBF4 in primary breast cancer and cancer cell lines (15). Also, inhibition of CDC7 in the cell lines can lead to stopping of the cell cycle. Thus, inhibition of CDC7 may be useful for the treatment of cancer $(15,16,18)$.

There is not any study that has been conducted on evaluation of the expression of CDC7 protein in the odontogenic cysts, so this study aimed to evaluate the immunohistochemial expression of this protein in the most common odontogenic cysts.

\section{MATERIALS AND METHODS}

In this cross-sectional study, 17 cases of $\mathrm{DC}, 18 \mathrm{OKC}$ and 13 $\mathrm{RC}$ were included. The samples were obtained from Oral and maxillofacial pathology department of Shiraz University of Medical Sciences (1998-2014). All samples had a definite diagnosis and an adequate epithelial component. Inflamed OKCs and DCs were excluded. Baseline data including the patients' age and gender as well as the location of the lesions were noted according to the patients' medical files.

IHC staining was performed using the Envision Labeled Peroxidase System (DAKO, Carpentaria, CA, USA). All the samples had been fixed in $10 \%$ formalin and embedded in paraffin previously. After deparaffinizationin xylene, the sections were dehydrated in alcohol and washed in distilled water. Antigen retrieval was done by DAKO cytomation target retrieval solution with $\mathrm{PH}=9$ during 20 minutes. Internal peroxidase activity was inhibited by $\mathrm{H}_{2} \mathrm{O}_{2} 3 \%$. We used CDC7 (Polyclonal rabbit antibody \# 1:50, Gentex, USA) as primary antibody. 3,3'-diaminobenzidinetetrahydrochloride (DAB) was used as chromogen and hematoxylin was applied for background staining.

Basal cell layer of the oral mucosa was used as positive control. For negative control, primary antibody was replaced by PBS (phosphate-buffered saline) solution. The cells with brown nuclei were considered as CDC7 positive. In high power, we counted at least 300 cells in three microscopic fields of each specimen. Samples with CDC7 staining $<5 \%, 5-10 \%$ and $>10 \%$ were considered as low, intermediate and high expression of $\mathrm{CDC7}$, respectively (19). Data were analyzed using SPSS software. ANOVA and Post-HOC tests were used. $\mathrm{P}$ value $\leq 0.05$ was considered significant statistically.

\section{RESULTS}

The mean \pm SD age of the cases was $30.66 \pm 10.25$ years old (range 12-54 years). Baseline characteristics regarding age, gender and location of the lesions in each group are shown in Table 1.

Nuclear expression of $\mathrm{CDC7}$ in basal and para-basal cell layers was observed in all specimens. In the DC group, the mean of CDC7 expression of cells was $3.47 \pm 1.4$ and it was limited to the basal cell layer (Fig. 1A). Also in RC, in addition to the basal cell layer, positive staining was observed in the parabasal layer and the mean of expression was 20.6 \pm 14.9 (Fig. 1B). In the OKC group, more positive CDC7 cells 
were observed in the parabasal layer and basal cell layer showed less staining. The mean of positive staining for OKC was $18.15 \pm 8.04$ (Fig. 1C).

According to ANOVA test, there was a statistically significant difference among the three groups in CDC7 expression $(\mathrm{P}<0.001)$. Post-HOC test showed that positive staining for $C D C 7$ in DC was lower than the two other cysts. ( $\mathrm{P}<0.001$ for both group); however, $\mathrm{RC}$ and OKC did not reveal any significant difference in staining for CDC7 $(P=0.738)$. CDC7 expression in the three groups was divided into three subgroups: low, medium and high, as illustrated in Table 3. Although most of the DCs showed low expression of $\mathrm{CDC7}$, the cases of $\mathrm{OKC}$ and $\mathrm{RC}$ revealed a high CDC7 expression rate.

\section{DISCUSSION}

In the present study, expression of CDC7 in the most common odontogenic cysts was evaluated. OKC had an aggressive behavior and tendency to high recurrence, but DC and $\mathrm{RC}$ showed slow growth and low recurrence. Although the exact mechanism for this difference is unknown, many studies explain that the epithelium of OKC could be involved in its aggressive behavior (2). However, some stromal components are also involved in this behavior (20). The results of this study showed that the expression of CDC7 in OKC and RC was higher than DC with a significant difference. In various studies on the expression of CDC7, the total conclusion was that the increase in the expression of this marker is related to higher proliferation activity, or increase in the aggressiveness of the tumor $(15,17,21$, 22). Its mechanism is phosphorylation of MCM2-7 complex (minichromosomal maintenance 2-7), activating the internal pathway of DNA-Helicase activity and initiating

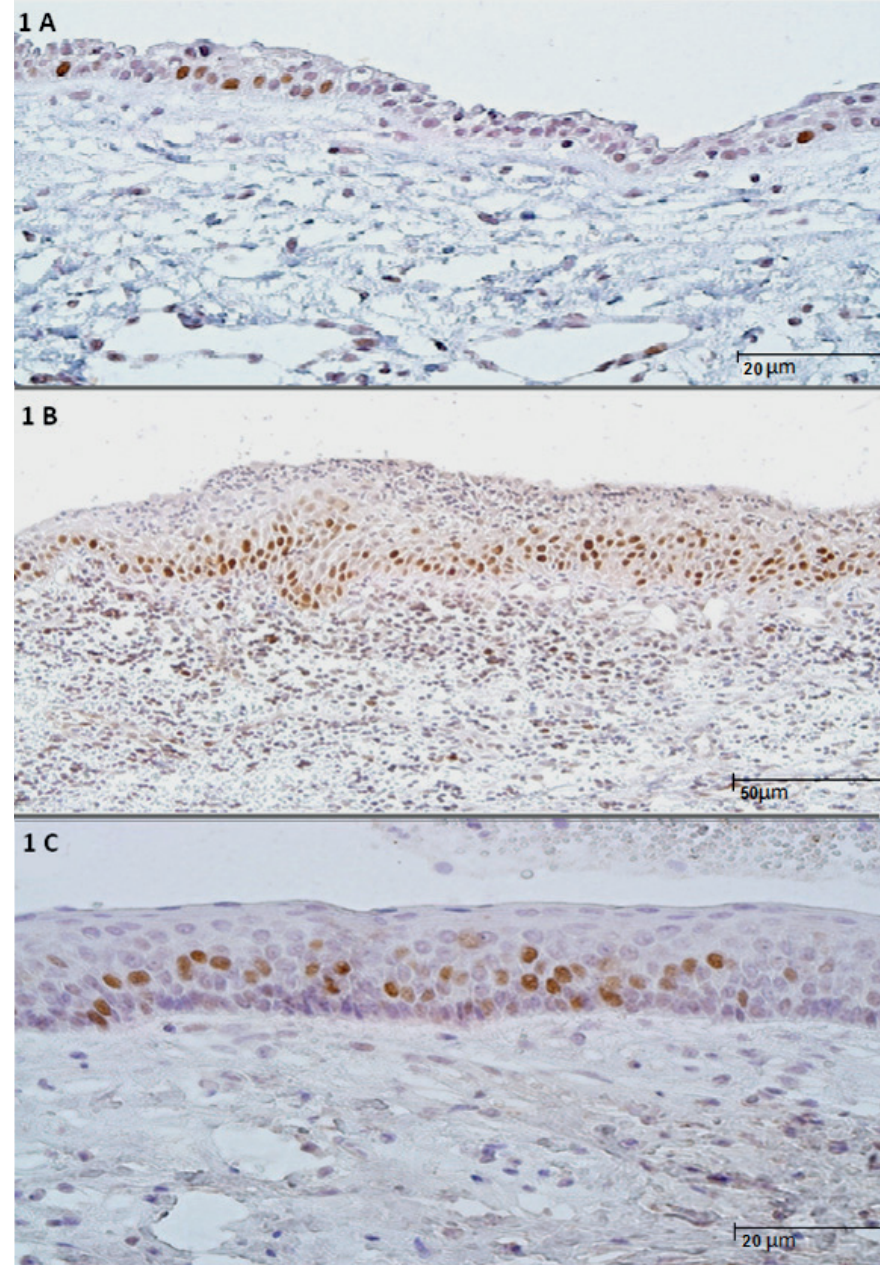

Fig. 1 Nuclear expression of CDC7; $1 \mathrm{~A}$ : in the basal cell layer of dentigerous cyst $(\times 400)$; $1 \mathrm{~B}$ : in the basal and parabasal classes of Radicular radicular cysts (×200); 1C: in the parabasal layer of odontogenic keratocyst $(\times 400)$.

Tab. 1 Baseline data of all study groups.

\begin{tabular}{|l|l|l|l|}
\hline Type of Cyst (N) & Age & Male : Female & Maxilla : Mandible \\
\hline Dentigerous Cyst (17) & $29.76 \pm 10$ & $14: 3$ & $7: 10$ \\
\hline Radicular Cyst (13) & $35 \pm 9.3$ & $8: 5$ & $8: 5$ \\
\hline Odontogenic Keratocyst (18) & $28.2 \pm 10.7$ & $8: 10$ & $4: 14$ \\
\hline
\end{tabular}

Tab. 2 CDC7 expression rate in all study groups.

\begin{tabular}{|l|l|l|l|}
\hline Type of Cyst (N) & CDC7 (Mean \pm SD) & Min-Max & P-value \\
\hline Dentigerous Cyst (17) & $3.5 \pm 1.4$ & $2-6.1$ & $0.000:$ vs Rad \\
\hline Radicular Cyst (13) & $20.6 \pm 14.9$ & $5.5-49$ & $0.000:$ vs OKC \\
\hline Odontogenic Keratocyst (18) & $18.1 \pm 8$ & \multirow{2}{*}{$9-32.6$} & $0.738:$ vs OKC \\
\hline
\end{tabular}

Tab. 3 Classification of CDC7 expression in the study.

\begin{tabular}{|l|l|l|l|}
\hline Type of Cyst(N) & Low & Moderate & igh \\
\hline Dentigerous Cyst(17) & 14 & 3 & - \\
\hline Radicular Cyst(13) & 1 & 3 & 9 \\
\hline Odontogenic Keratocyst (18) & - & 1 & 17 \\
\hline
\end{tabular}


the replication of DNA (23); thus, it results in an increase in cell proliferation (24). In previous studies, an increase of MCMs such as MCM2 and MCM 3 was observed in OKC (25); also, many studies have shown an increased proliferative activity of this cyst rather than other odontogenic cysts. MCM positive cells have a high CDC7 activity (25).

$C D C 7$ is used as an initiator for DNA replication in anti-cancer therapeutic purposes. Inhibition of CDC7 expression can cause a destructive $S$ phase and ability to destroy the cancer cells. This feature was recognized by a G1 reverse phase, during inhibition of CDC7 expression that happened by reactivation of the cell cycle checkpoints which were damaged due to cancer (25). In normal cells, a p53-dependent pathway actively inhibits the damaged $S$ phase during the absence of CDC7 kinase, and p53 is essential for maintenance of check-points of the cell cycle (16). Our results reflect the role of CDC7 in higher proliferation of OKC. Moreover, the expression of CDC7 in OKC was seen mostly in the parabasal layer, whereas in other groups it was observed in the basal cell layer. Many studies have observed the suprabasal layer of OKC as the proliferative layer, unlike other odontogenic cysts. In a study, it has been mentioned that the biologic behavior of OKC is due to its proliferative parabasal layer in which Ki-67, p53 and p63 express highly (24).

In this study, we found a higher expression of CDC7 in $\mathrm{RC}$; as proliferation of the RC cells is attributed to the inflammatory process stimuli, we raise the question whether the high CDC7 imunoexpression observed in RC might be related to the significant inflammation. Guler and colleagues studied the expression of Ki67 and MCM2 factors, as well as the effects of inflammation on the markers of cell cycle in the cases of dental follicle and odontogenic cysts such as RC, DC and OKC. They concluded that the expression of Ki67 in dental follicle and OKC and expression of MCM2 in RC and OKC were higher statistically. The result of MCM2 in the mentioned study is similar to that of CDC7 expression in our study, which was higher in RC and OKC. They mentioned that there could be a relationship between higher inflammation in RC and higher expression of MCM2 proliferation marker (26).

Among the study groups, DC and OKC showed uniformity in the expression of CDC7; as $82 \%$ of DC showed low expression and $94 \%$ of OKC revealed high expression of CDC7, but RC had a more scattered staining pattern. Based on these results, the expression of CDC7 could be affected by the amount and type of inflammatory cells. Given that the proliferative markers are used to make a differential diagnosis of pathologic lesions, based on these results, it seems that the effectiveness of this marker in making differential diagnosis in inflamed odontogenic cysts is low. However, further studies focusing on the impact of inflammation are suggested for confirming this point.

\section{CONCLUSION}

Based on the present findings, it is concluded that there is a higher expression of CDC7 in odontogenic cysts with higher proliferative activity and higher aggressiveness, such as OKC. Therefore, it could be a potential prognostic marker. Since the expression of this marker was also high in RC, inflammation appears to contribute to CDC7expression.

We suggest further studies comparing the $\mathrm{RC}$ with inflamed DC and OKC to evaluate the effectiveness of this marker in diagnosis of inflamed lesions and a larger sample size to get a cut-off point for differential diagnosis of the odontogenic cyst.

\section{ACKNOWLEDGEMENTS}

The authors thank the Vice-chancellery of Shiraz University of Medical Sciences for supporting this research (Grant \#10085). This manuscript is based on the undergraduate thesis of Ahmad Alipour Tuyeh. The authors are also grateful to Dr. Salehi in Dental Research Development Center of Shiraz Dental School for the statistical analysis. The authors would like to thank the research consultation center (RCC) of Shiraz University of Medical Sciences for their invaluable assistance in editing this article.

\section{FUNDING}

Vice-chancellery of Shiraz University of Medical Sciences supported this research (Grant \#10085).

\section{CONFLICT OF INTEREST}

\section{None}

\section{ETHICAL APPROVAL}

This article has been approved by Ethic committee of Shiraz University of Medical Sciences (Code: IR.SUMS. REC.1394.S762).

\section{REFERENCES}

1. Khalifa GA, Shokier H, Abo-Hager E. Evaluation of neoplastic nature of keratocystic odontogenic tumor versus ameloblastoma. Journal of the Egyptian National Cancer Institute 2010; 22(1): 61-72.

2. Swetha P, Ramesh K, Madhavan N, Veeravarmal V, Sameera A. Expression of inducible nitric oxide synthase in the epithelial linings of odontogenic keratocyst, dentigerous cyst and radicular cyst: a pathological insight. Ann Med Health Sci Res. 2014; 4(4): 583-9.

3. Selvamani M, Donoghue M, Basandi PS. Analysis of 153 cases of odontogenic cysts in a South Indian sample population: a retrospective study over a decade. Brazilian Oral Research 2012; 26(4): 330-4.

4. Bhardwaj B, Sharma S, Chitlangia P, Agarwal P, Bhamboo A, Rastogi K. Mandibular Dentigerous Cyst in a 10-Year-Old Child. International Journal of Clinical Pediatric Dentistry 2016; 9(3): 281-284.

5. Mohan KR, Natarajan B, Mani S, Sahuthullah YA. An infected dentigerous cyst associated with an impacted permanent maxillary canine,inverted mesiodens and impacted supernumerary teeth. J Pharm Bioallied Sci 2013; 5: 135-8.

6. Razavi SM, Khalesi S, Torabinia N. Investigation of clinicopathological parameters alongside with p53 expression in primary and recurrent keratocysticodontogenic tumours. The Malaysian Journal of Pathology 2014; 36(2): 105.

7. Bande CR, Prashant M, Sumbh B, Pandilwar P. Prevalence, treatment and recurrence of odontogenic keratocyst in central India. Journal of Maxillofacial and Oral Surgery 2010; 9(2): 146-9. 
8. Madras J, Lapointe H. Keratocystic odontogenic tumour: reclassification of the odontogenic keratocyst from cyst to tumour. Texas Dental Journal 2008; $125(5): 446$.

9. Speight P, Devillers P, Li T-J, Odell EW, Wright JM. Odontogenic Keratocyst. In: El-Nagger AK, Ohan JKC, Grandis JR, Takata T, Slootweg PJ, eds. WHO Classifiaction of Head and Neck Tumours WHO/IARC Clasifiaction of Tumors, 4th Edition, Volume 9, 2017, pp. 235-236.

10. Vidhale G, Jain D, Jain S, Godhane AV, Pawar GR. Management of Radicular Cyst Using Platelet-Rich Fibrin \& Iliac Bone Graft - A Case Report. J Clin Diag Res. 2015; 9(6); ZD34-ZD36.

11. Omoregie F, Sede M, Ojo A. Ameloblastomatous change in radicular cyst of the jaw in a Nigerian population. Ghana Med J 2015; 49(2): 107-11.

12. Lamba G, Ravi G. Replantation of Displaced Underlying Successor and Marsupialization of Radicular Cyst associated with a Primary Molar. International Journal of Clinical Pediatric Dentistry 2015; 8(1): 70.

13. Bava FA, Umar D, Bahseer B, Baroudi K. Bilateral Radicular Cyst in Mandible: An Unusual Case Report. Journal of International Oral Health: JIOH 2015; 7(2): 61.

14. Tenca P, Brotherton D, Montagnoli A, Rainoldi S, Albanese C, Santocanale C. CDC7 is an active kinase in human cancer cells undergoing replication stress. Journal of Biological Chemistry 2007; 282(1): 208-15.

15. Bonte D, Lindvall C, Liu H, Dykema K, Furge K, Weinreich M. CDC7Dbf4 kinase overexpression in multiple cancers and tumor cell lines is correlated with p53 inactivation. Neoplasia 2008; 10(9): 920-31.

16. Li W, Zhao XL, Shang SQ, Shen HQ, Chen X. Dual Inhibition of Cdc7 and Cdk9 by PHA-767491 Suppresses Hepatocarcinoma Synergistically with 5-Fluorouracil. Curr Cancer Drug Targets 2015; 15(3): 196-204.

17. Hess GF, Drong RF, Weiland KL, Slightom JL, Sclafani RA, Hollings- worth RE. A human homolog ofthe yeast CDC7 gene is overexpressed in some tumors and transformed cell lines. Gene 1998; 211(1): 13340.

18. Dally RD, Woods TA. CDC7 inhibitors. Google Patents; CA2900773A1. 2014.

19. Clarke LE, Fountaine TJ, Hennessy J, et al. CDC7 expression in melanomas, Spitz tumors and melanocytic nevi. Journal of Cutaneous Pathology 2009; 36(4): 433-8.

20. Tekkesin MS, Mutlu S, Olgac V. The role of RANK/RANKL/OPG signalling pathways in osteoclastogenesis in odontogenic keratocysts, radicular cysts, and ameloblastomas. Head Neck Pathol 2011; 5(3): 248-53.

21. Kulkarni AA, Kingsbury SR, Tudzarova S, et al. CDC7 kinase is a predictor of survival and a novel therapeutic target in epithelial ovarian carcinoma. Clinical Cancer Research 2009; 15(7): 417-25.

22. Shi N, Xie W-B, Chen S-Y. Cell division cycle 7 is a novel regulator of transforming growth factor- $\beta$-induced smooth muscle cell differentiation. Journal of Biological Chemistry 2012; 287(9): 6860-7.

23. Seyedmajidi $M$, Shafaee $S$, Siadati $S$, et al. Immunohistochemical analysis of COX-2 expression in dentigerous cyst, keratocystic odontogenic tumor and ameloblastoma: A comparative study. Dent Res J (Isfahan) 2015 May-Jun; 12(3): 278-84.

24. Gurgel CAS, Ramos EAG, Azevedo RA, Sarmento VA, da Silva Carvalho AM, dos Santos JN. Expressionof Ki-67, p53 and p63 proteins in keratocyst odontogenic tumours: an immunohistochemical study. Journal of Molecular Histology 2008; 39(3): 311-6.

25. Sainsbury R, Proctor I, Rodriguez S, et al. Targeting DNA replication before it starts: Cdc7 as a therapeutic target in p53 mutant Her2 and triple negative breast cancer. Breast Cancer Research 2010; 12: 50.

26. Güler N, Comunoğlu N, Cabbar F. Ki-67 and MCM-2 in Dental Follicle and Odontogenic Cysts: The Effects of Inflammation on Proliferative Markers. The Scientific World Journal 2012; 2012: 946060. 